\title{
COLOQUIO INTERNACIONAL SOBRE CRECIMIENTO URBANO Y PROBLEMAS DEL AGUA (RABAT, OCTUBRE 1988)
}

Por

MATEO MARCO

El Centro de Viena inició en 1984 un programa de investigación sobre «El agua y los hombres en los países del Mediterráneo y del Mar Negro». En España, dentro de este proyecto, se han realizado tres congresos: uno sobre "Agua y poblamiento musulmán» (Benissa, abril 1987), otro sobre "Avenidas fluviales e inundaciones en la cuenca del Mediterráneo" (Alicante-Murcia, mayo 1988 ) y un tercero sobre "La ciudad islámica" (Zaragoza, diciembre 1988).

En consonancia con este proyecto general se celebró un coloquio internacional en Rabat, desde el 20 al 22 de octubre de 1988, organizado por el Centro de Viena y I'Institut Agronomique et Vétérinaire Hassan II, sobre "Croissance urbaine et problèmes de l'eau dans les pays du bassin méditerranéen et de la Mer Noire». En este coloquio internacional se analizaron, principalmente, los problemas urbanos de abastecimiento de agua centrándose, algunos trabajos, en situaciones surgidas a raíz del desarrollo turístico.

Enumeramos algunas de las comunicaciones presentadas que trataron temáticas árabes:

AZZAM, Y.: Les problemes urbains dans le rapports nil - Le Caire.

BENAMEUR, M.: Besoins en eau des villes marocaines.

BERRADA, A.: Alimentation de la Wilaya du grand Casablanca en eau potable a court, moyen et long terme.

BOULIFA, A.: Le monopole de Tanger sur les eaux de sa region: consequences sur l'espace rural environnant. 
EL ABDELLAOUI, M.: Le reseau «skundu»: Ancien systeme d'approvisionnement de la Medina de Tetouan en eau potable.

EL CHEIKH, M. I.: Croissance spectaculaire urbaine et demographique et problemes de l'eau potable au Kuwait (Arabie).

FEJJAL, A.: Les problemes d'approvisionnement des fes en eau.

HAYDER, A.: Le probleme de l'eau a gabes: Gestion conflictuelle et etatisation.

JENNAN, L. ET MARTIN, J.: L'eau et la ville au pied du moyen atlas: les cas de Sefrou, Azrou et Khenifra.

JOLE, M.: Rapports service-usagers a partir d'une politique recente de branchement generalise: Le cas de Kariat-Oulad Moussa, a Rabat-Sale et les branchements sociaux (1).

LACOSTE, X.: D'une consommation des ressources locales a la mise en place d'un systeme articulant gestion nationale de la production d'eau et gestion locale de la distribution: le cas de Rabat au Maroc.

MAGAGNOSC, J. S. - TOUBACHE, H.: L'emprise des villes algeriennes sur les espaces hydrauliques: Le cas d'Oran. Consequences sur la politique d'amenagement du territoire.

MARCO, M.: Bibliographie sur la maitrise des eaux en Espagne a l'epoque islamique.

MEBARKI, A.: Alimentation en eau de la ville de Constantine (Algerie): Bilan ressources-besoins et perspectives d'amenagement.

OMRANE, M.: La croissance urbaine de Tunis et la question de l'eau.

PERENNES, J. J.: Regards d'un economiste sur l'evolution de la notion de rarete de l'eau au Maghreb.

SEFRIOUI, F.: Economie des ressources en eau et croissance urbaine: Le cas de Meknes.

SETHOM, H.: Les dangers de la priorite absolue aux villes dans la repartion de l'eau disponible et Tunisie. 